\title{
High-dose-rate brachytherapy in severe trismus: Making it happen!
}

\author{
Kanchan P. Dholam, MDS', Gurkaran Preet Singh, MDSl, Sarbani Ghosh Laskar, MD², Sandeep V. Gurav, MDSl, \\ Gorakh S. Ahire, QDT' \\ 'Department of Dental and Prosthetic Surgery, Tata Memorial Hospital, HBNI, Mumbai, ${ }^{2}$ Department of Radiation Oncology. Tata Memorial \\ Hospital, HBNI, Mumbai, India
}

\begin{abstract}
Brachytherapy has been widely employed as a salvage or adjuvant modality in localized early and/or recurrent lesions. In recent years, advances in brachytherapy techniques have helped to achieve better loco-regional disease control and higher survival rates at the cost of limited morbidity. This is mainly owing to the development of technologically advanced three-dimensional computer planning systems and treatment delivery techniques. Low-doserate brachytherapy has been substituted by high-dose-rate and pulsed-dose-rate techniques, which allow better dose optimization. Inter-disciplinary approach results in fabrication of customized intra-oral surface mould, which allows accurate dose delivery, excellent dose distribution, and is less time-consuming. However, fabrication of surface mould becomes extremely challenging when intra-oral anatomic factors are unfavorable. We present a report on the management of a previously-irradiated completely edentulous patient with severe trismus for whom high-dose-rate surface mould brachytherapy had been prescribed. A unique, reliable, and practical solution has been presented based firmly on the scientific knowledge of contemporary implant dentistry.

Key words: dental implant, head-neck cancer, surface mould, trismus, tumor recurrence.

\section{Purpose}

Brachytherapy has been widely employed as a salvage or definitive modality in recurrent and/or localized early lesions, respectively [1]. In recent years, with the evolution of new techniques, high-dose-rate brachytherapy (HDR-BT) is usually preferred as it is an outpatient-based procedure, which does not require hospitalization and reduces radiation safety concerns [2,3]. For sites within head and neck region like oral cavity and oropharynx, brachytherapy provides an effective alternative especially when surgery is morbid or not possible [4]. Effective inter-disciplinary approach helps in fabrication of customized radiation-carrier prosthesis, which enables accurate dose delivery with excellent dose distribution [2].

However, fabrication of surface mould becomes extremely challenging when intra-oral anatomic factors are unfavorable. We present a report on the management of a previously-irradiated completely edentulous patient with severe trismus utilizing surface mould-based HDR-BT.

\section{Case report}

A 62-year-old male patient reported with a non-healing ulcer on the roof of mouth on left side. The ulcer ap- peared 2 months back and it was sudden in onset, associated with occasional bleeding.

\section{Examination}

Intra-oral examination revealed $1.5 \mathrm{~cm} \times 1.5 \mathrm{~cm} \mathrm{su}-$ perficial ulcer on left side of hard palate extending till the junction of hard and soft palate posteriorly, and edentulous alveolar ridge laterally (Figure 1). Patient was completely edentulous in upper and lower arches, with severe trismus and maximal mouth opening of $1 \mathrm{~cm}$. Right hemi-mandible was absent and reconstructed with pectoralis major myocutaneous (PMMC) flap. There was a mandibular deviation with PMMC flap obscuring palate on right side. Antero-inferiorly, the flap was extending till right oral commissure leading to microstomia. It showed signs of sub-cutaneous stricture formation and fibrosis, thus compromising orofacial musculature (Figure 2). Neck examination did not reveal evidence of cervical adenopathy.

\section{Previous medical history}

Patient had been diagnosed with moderately differentiated squamous cell carcinoma (MDSCC) of the lip with 
synchronous well-differentiated squamous cell carcinoma (WDSCC) of the tongue in 2005. He had undergone wide excision of upper lip-buccal mucosa and tongue followed by adjuvant external beam radiotherapy (EBRT) to face and neck to a total dose of 50 Gy/ 25 fractions till July 2005. He developed recurrence and was diagnosed with WDSCC of the tongue in 2008. He underwent wide local excision and commisuroplasty but did not receive any adjuvant radiotherapy or chemotherapy. In 2012, the patient presented with second primary lesion (MDSCC) involving the right buccal mucosa, extending till the mandible. He underwent extended right hemi-mandibulectomy and modified neck dissection followed by reconstruction with PMMC flap. He received further adjuvant EBRT to face and neck to a total dose of $60 \mathrm{~Gy} / 30$ fractions till August 2012. Subsequently, he was lost to follow-up and did not report to the hospital till 2016.

\section{Investigations}

Histopathologic examination of palatal ulcerative lesion revealed fragments of hyperplastic squamous epithelium with focal areas of dysplasia compatible with squamous carcinoma. Contrast-enhanced computed topographical (CECT) examination did not reveal erosion of underlying left maxilla (Figure 3). Similarly, 18-fluorodesoxyglucose positron-emitted topographical scan (FDG PET) did not reveal any abnormal focal increased tracer uptake in head and neck region or elsewhere in the body.

\section{Management}

In view of morbidity of previous surgeries and radiotherapy, salvage re-surgery and EBRT were not feasible. HDR brachytherapy was prescribed keeping in mind superficial nature of disease and long disease-free interval (DFI). However, fabrication of conventional surface mould was considered improbable due to completely edentulous maxilla coupled with severe trismus and microstomia.

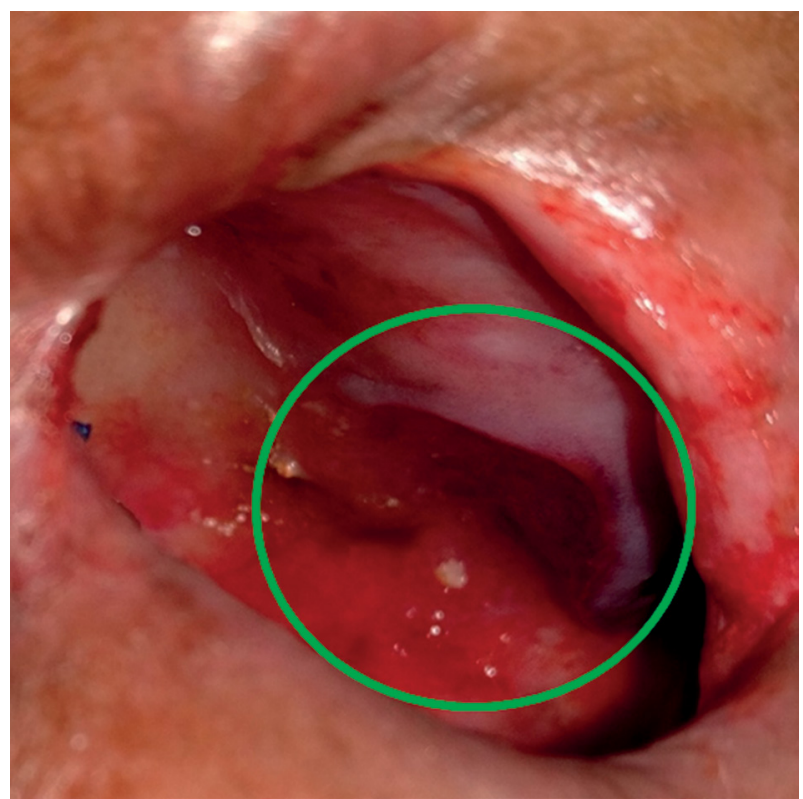

Fig. 1. Intra-oral presentation of a $1.5 \mathrm{~cm} \times 1.5 \mathrm{~cm}$ superficial ulcer on the left side of hard palate

An alternate mode of retention in the form of dental implant was proposed for surface mould. An orthopantograph (OPG) examination showed adequate bone depth in left maxillary premolar-molar region. Primary impression was made with irreversible hydrocolloid material (Zelgan Plus, Dentsply ${ }^{\mathrm{TM}}$ ). Custom tray was constructed and subsequently, muscle trimming was carried out using low-fusing impression compound $\left(\mathrm{DPI}^{\mathrm{TM}}\right.$ Pinnacle tracing stick) to accurately register the borders of the surface mould. A corrective wash impression was made using medium body elastomeric impression material (Aquasil impression paste, Dentsply ${ }^{\mathrm{TM}}$ ) (Figure 4) to obtain master cast, and customized surface mould was fabricated using heat-polymerized acrylic resin.
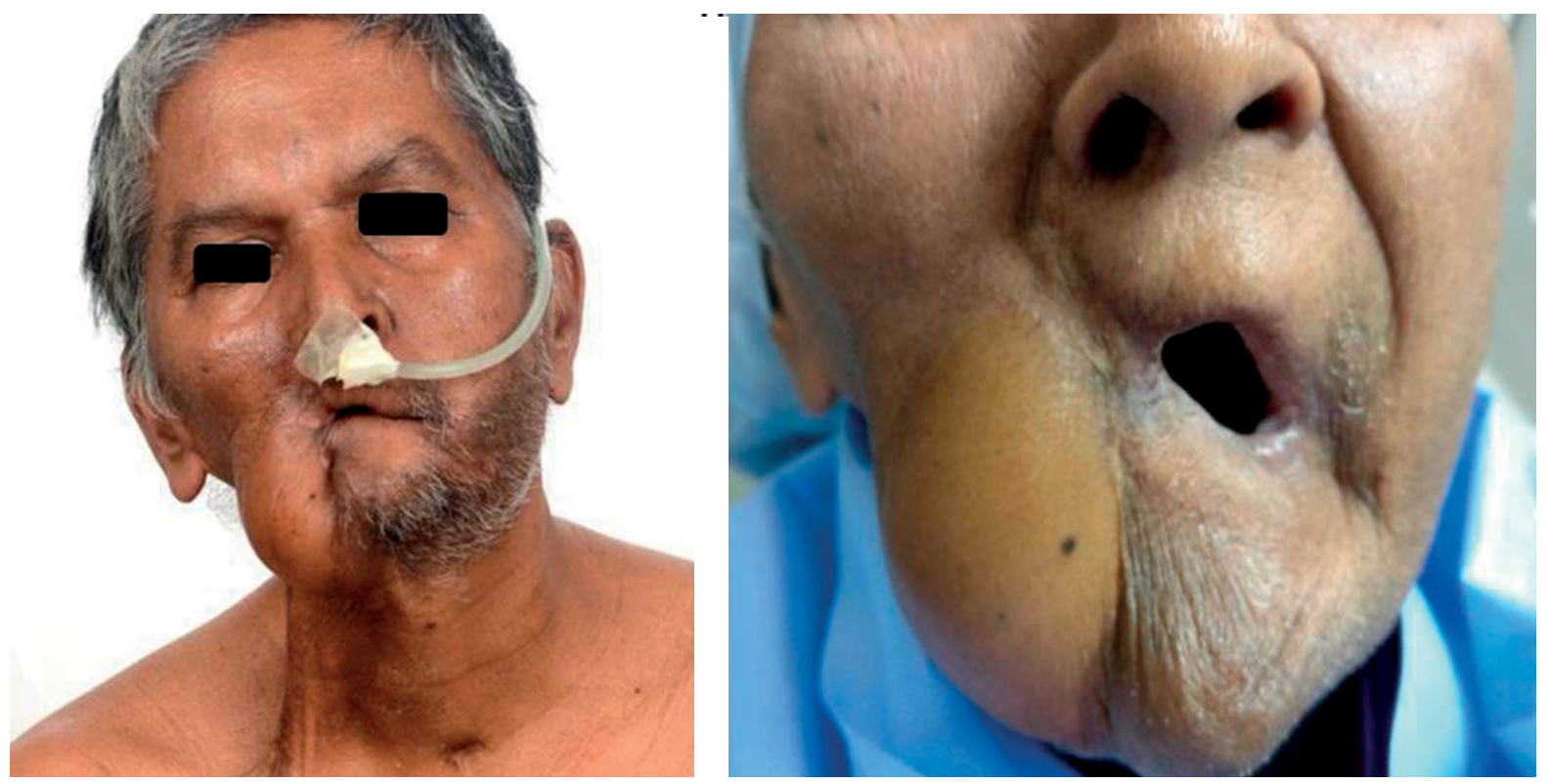

Fig. 2. Clinical challenge of severe trismus, edentulous maxilla and microstomia 


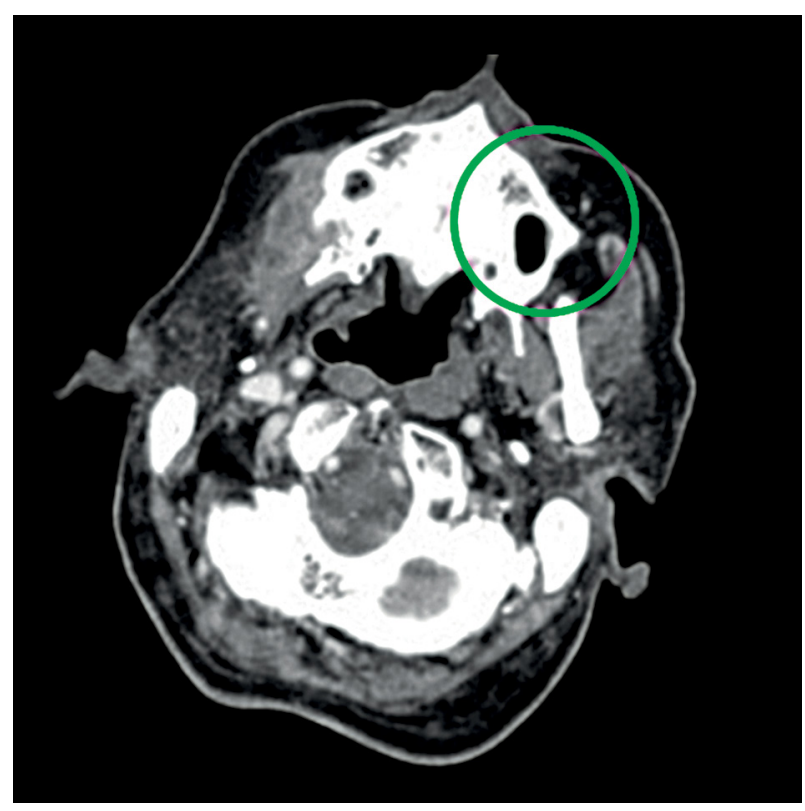

Fig. 3. Contrast-enhanced computed tomography scan showing no underlying bone erosion

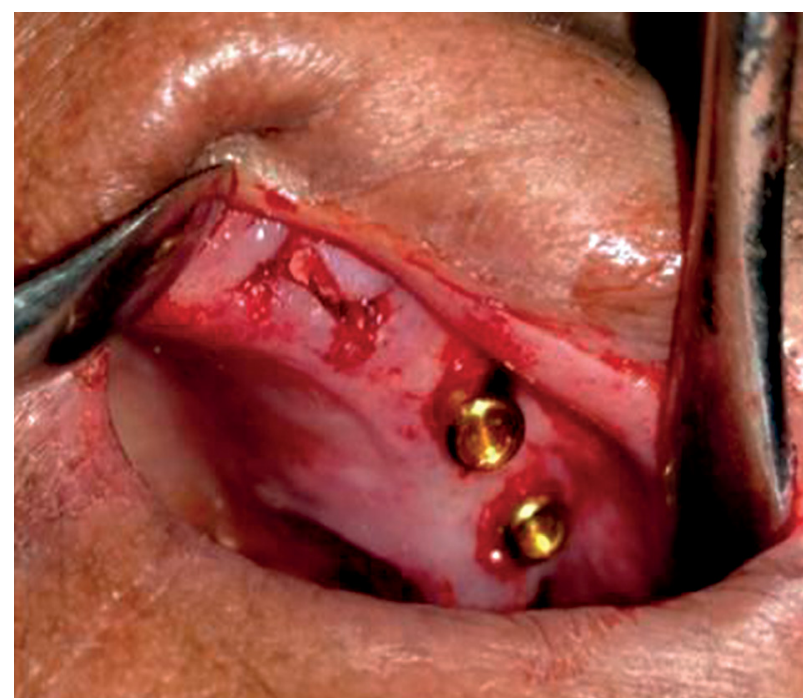

Fig. 5. Flapless placement of dental implants with locator abutments

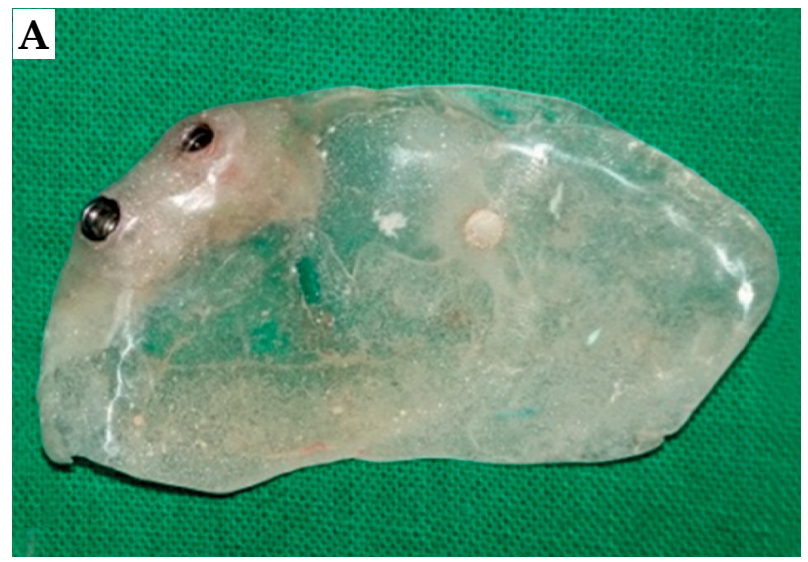

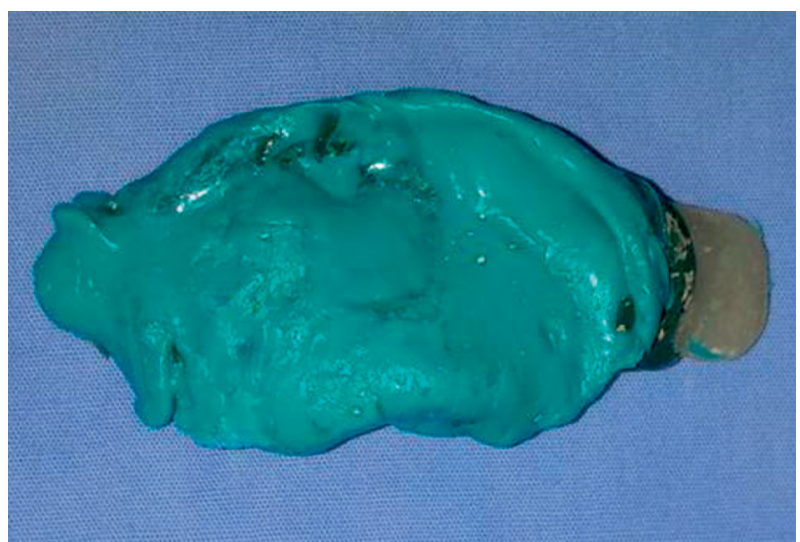

Fig. 4. Definitive impression for fabrication of surface mould

Subsequently, two Nobel Active ${ }^{\mathrm{TM}}$ (Nobel Biocare ${ }^{\mathrm{TM}}$ ) implants each measuring $3.5 \mathrm{~mm} \times 8 \mathrm{~mm}$ were placed in left maxillary $1^{\text {st }}$ and $2^{\text {nd }}$ pre-molar region, using flapless technique under local anesthesia and antibiotic cover. Locator $^{\mathrm{TM}}$ abutments (Nobel Biocare ${ }^{\mathrm{TM}}$ ) were placed (Figure 5), and implants were immediately loaded with radiation-carrier prosthesis (Figures 6A-B).

Primary healing was uneventful and CT scan was carried out after 24-48 hours to verify intimate fit of prosthesis. Once satisfactory, surface mould was removed and softened modeling wax was adapted onto the polished surface. Four 6-F nylon catheters were placed $1 \mathrm{~cm}$ apart and secured in wax (Figure 7). Radiation simulation was carried out using Oncentra ${ }^{\circledR}$ Brachyplanning software (Elekta technologies). The reference isodose was chosen based on coverage of a predetermined target volume. Subsequently, remote after-loading ${ }^{91}$ Ir HDR brachytherapy, 3.5 Gy twice daily fractions minimum 6 hours apart were administered to a total dose of 49 Gy over 7 days (Figure 8). The patient was put on nasogastric feed to prevent any untoward masticatory force on the immediately loaded implants. On completion of radiotherapy, the prosthesis was removed, and locator abutments were replaced with cover screws and implants were submerged under oral mucosa.

Patient has been on regular follow-up and exhibited good loco-regional control at the end of six months (Figure 9).

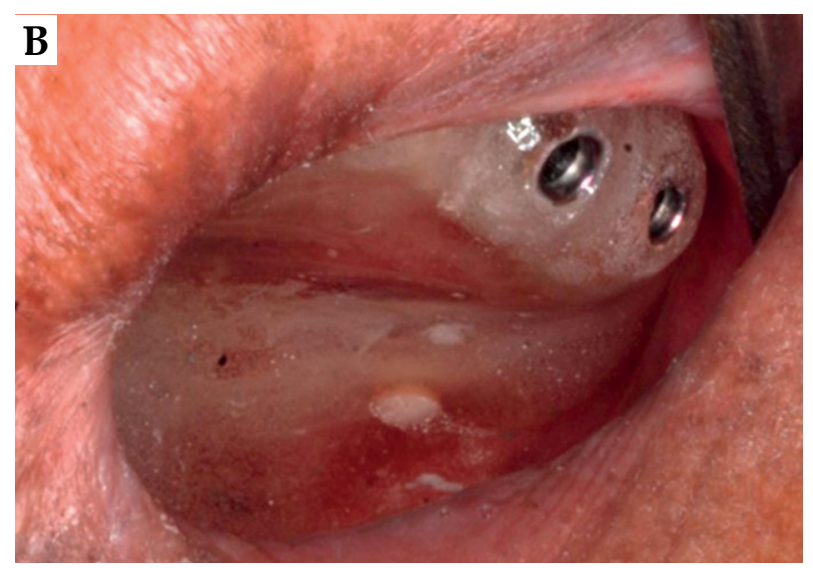

Fig. 6. Radiation carrier prosthesis fabricated (A) and placed in-situ (B) 

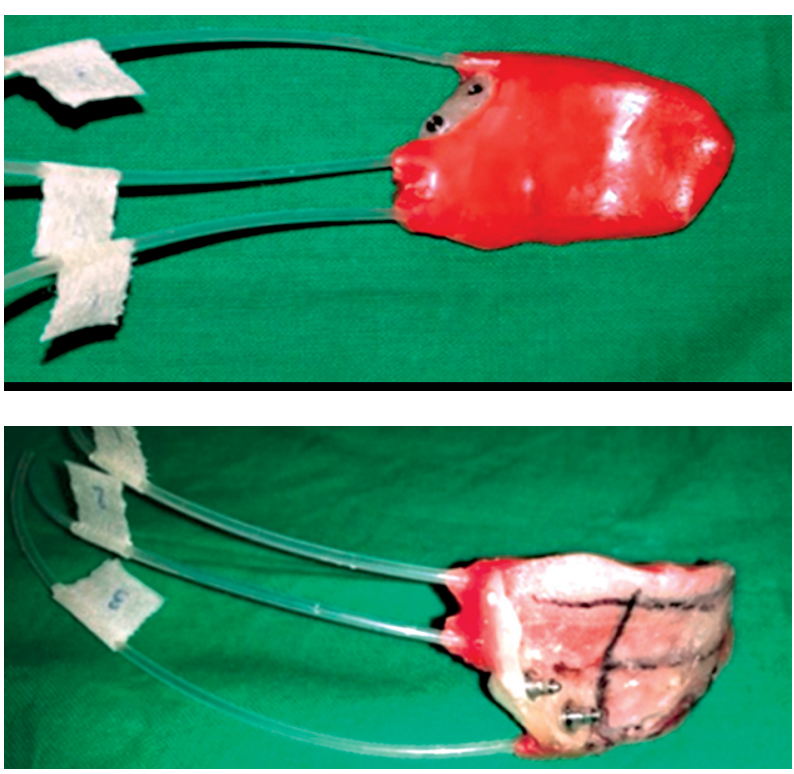

Fig. 7. Surface mould preparation

\section{Discussion}

Loco-regional recurrence is the predominant cause for failure in locally advanced head and neck region [5]. Surgery is the most preferred salvage modality; although majority of patients are ineligible for the same due to presence of co-morbid conditions, advanced local spread, and/or morbidity of previous curative therapy [6]. EBRT in an area previously radiated with high-dose CTRT carries a significant risk of associated long-term toxicities/ morbidities [1]. Re-radiation damages critical structures in the head and neck region such as the salivary glands, the mandible, and masticatory muscles, which sustain undesirable late effects [7]. De Crevoisier et al. have reported significant late toxicities including trismus 30\%, mucosal necrosis $21 \%$, and osteoradionecrosis $8 \%$ in a large study consisting of 169 patients, thus highlighting the peril of prescribing EBRT in previously radiated tissues [6].

Brachytherapy alone or in combination with external beam (EBRT), and/or chemotherapy leads to local dose escalation, rapid fall-off, and improved outcomes in recurrent head-and-neck cancer setting [8]. Technologically advanced three-dimensional (3D) computer planning and optimization allow better dose-distribution by varying dwell times through HDR systems [9]. Hot and cold spots are minimized, thereby maximizing tumor control, and preserving organs-at-risk. Ciervide et al. have studied the use of intraoral mould-based HDR brachytherapy to treat tumors involving the maxillary antrum and have observed a dose reduction of $4-43 \%$ in organs-at-risk (OAR) [10].

In the current report, in view of morbidity of previous surgeries and radiotherapy, a treatment plan comprising of remote afterload HDR brachytherapy was prescribed. This is in compliance with recent GEC-ESTRO recommendations for head and neck brachytherapy in squamous cell carcinomas [1].

Since HDR brachytherapy involves short treatment time, it is imperative that catheters remain in the exact position as

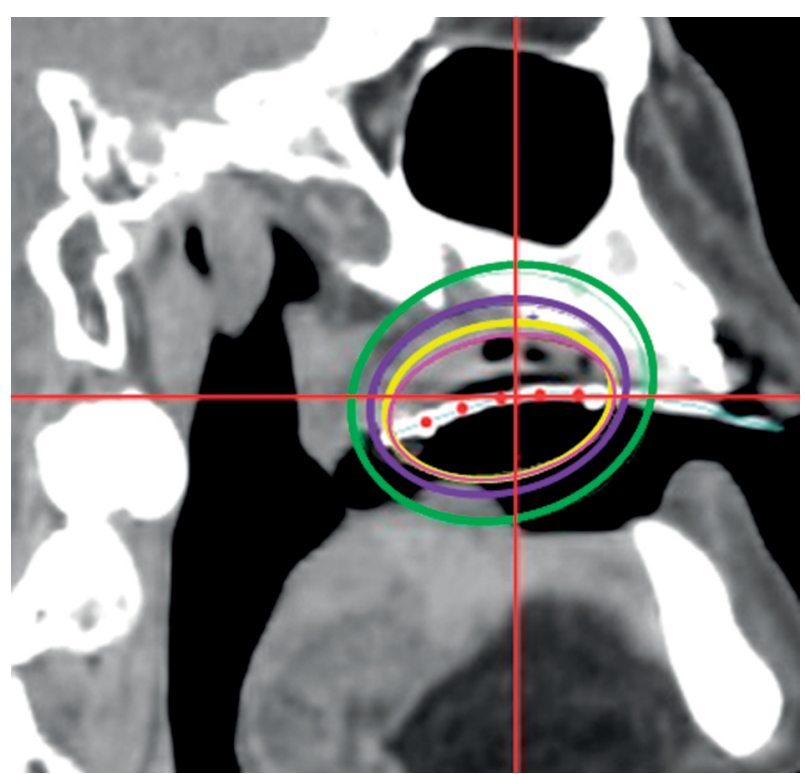

Fig. 8. High-dose-rate brachytherapy isodose curves for $3.5 \mathrm{~Gy} / \mathrm{BD}$ for 7 days to a total dose of $49 \mathrm{~Gy}$. Red zone: $200 \%$ dose (7 Gy), yellow zone: $150 \%$ dose (5.25 Gy), purple zone: $100 \%$ dose $(3.5 \mathrm{~Gy})$, and green zone: $50 \%$ dose $(1.75 \mathrm{~Gy})$

determined by the plan. Hence, customized mould was designed to provide adequate dose coverage to tumor volume.

However, fabrication of surface mould was challenging due to unfavorable anatomic factors and absence of teeth for retention. Consequently, implant-retained radiation-carrier prosthesis was fabricated. These fixtures served as retentive pillars for prosthesis and provided adequate stability and support.

Additionally, TiUnite surface of Nobel Active ${ }^{\mathrm{TM}}$ implants along with unique implant body and thread design enabled immediate loading of these fixtures with corresponding locator (Nobel Biocare ${ }^{\mathrm{TM}}$ ) abutments [11]. Thus, surface mould was immediately secured onto these fixtures, which helped in prompt initiation of salvage HDR brachytherapy after appropriate planning.

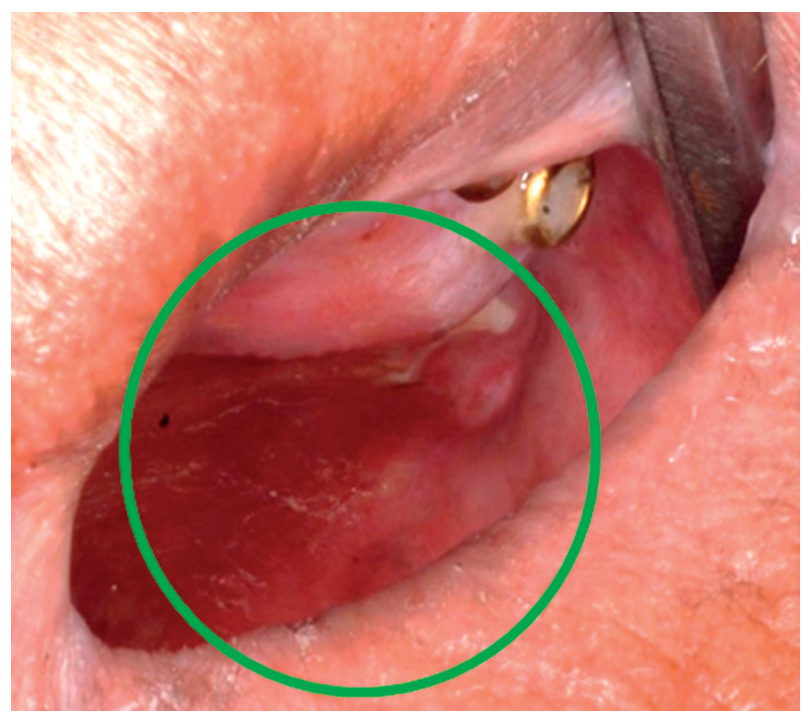

Fig. 9. Six-month post-operative result 
Since there is a lack of randomized trials on the use of mould-based HDR brachytherapy [12], vital parameters such as optimal time, dose, and fractionation were decided based on our institute experience and consensus statement given in GEC-ESTRO recommendations.

Nishimura et al. [13], Obinata et al. [14], Kudoh et al. [15], Mukherji et al. [2], and Chatani et al. [16] have presented their experience with the use of customized surface mould-based HDR brachytherapy, and concluded that it is safe and efficient for early and superficial lip and buccal mucosa lesions. Matsuzaki et al. [17] have defined HDR-BT as a viable treatment option, especially in situations when other therapeutic modalities are limited by age, performance status, and other factors. Budrukkar et al. [18] studied the clinical outcomes with high-doserate surface mould brachytherapy in thirty-five patients with malignant localized early T1/T2, N0 intra-oral and skin malignancies involving head and neck region. They reported excellent 5-year local control of $94.2 \%$ and $68.2 \%$ for T1 and T2, respectively. Bhalavat et al. [19] also verified the role of HDR-BT in head-and-neck cancer as a proven, effective, and safe treatment method with excellent long-term outcome.

Application of CAD/CAM technology and 3D printing has also shown promising results in the fabrication of well-fitting, superior quality surface mould prosthesis. Initial reports have demonstrated highly accurate dose delivery and improved treatment outcome [20].

We report a novel and predictable method of fabrication of surface mould in a challenging intra-oral scenario. Our technique relies on scientifically-proven methodology to deliver an accurate and effective result in a patient who was not suited for any other therapeutic modality. Our case report indicates that often, in a patient with recurrent head and neck cancer, an out-of-the-box approach has to be applied to execute a well-established standard of care.

\section{Conclusions}

Re-irradiation of recurrent head and neck cancer is a therapeutic challenge. We consider our technique of fabrication of surface mould to be an appropriate treatment option for completely edentulous patients with recurrent oral cavity carcinoma, for whom salvage modalities are limited due to age, morbidity of previous radiotherapy, severe trismus, or microstomia. A unique, reliable and practical solution has been presented based firmly on the scientific knowledge of contemporary implantology.

\section{Disclosure}

The authors report no conflict of interest.

\section{References}

1. Kovács G, Martinez-Monge R, Budrukkar A et al. GECESTRO Head \& Neck Working Group. GEC-ESTRO ACROP recommendations for head \& neck brachytherapy in squamous cell carcinomas: $1^{\text {st }}$ update - Improvement by cross sectional imaging based treatment planning and stepping source technology. Radiother Oncol 2017; 122: 248-254.
2. Mukherji A, Mourougan S, Saravannan K et al. Dosimetric analysis and clinical outcomes in CT-based mould brachytherapy in early oral cancers in patients unfit for surgery. J Contemp Brachytherapy 2015; 7: 147-153.

3. Narayana A, Cohen GN, Zaider M et al. High-dose-rate interstitial brachytherapy in recurrent and previously irradiated head and neck cancers - preliminary results. Brachytherapy 2007; 6: 157-163.

4. Hepel JT, Syed AM, Puthawala A et al. Salvage high-doserate (HDR) brachytherapy for recurrent head-and-neck cancer. Int J Radiat Oncol Biol Phys 2005; 62: 1444-1450.

5. Due AK, Vogelius IR, Aznar MC et al. Recurrences after intensity modulated radiotherapy for head and neck squamous cell carcinoma more likely originate from regions with high baseline 18F-FDG uptake. Radiother Oncol 2014; 111: 360-365.

6. De Crevoisier R, Bourhis J, Domenge C et al. Full dose reirradiation for unresectable head and neck carcinoma. Experience at the Gustave Roussy Institute in a series of 1659 patients. J Clin Oncol 1998; 16: 3556-3562.

7. Haraf D, Weichselbaum R, Vokes E. Re-irradiation with concomitant chemotherapy of unresectable recurrent head and neck cancer: A potentially curable disease. Ann Oncol 1996; 7: 913-918.

8. Gerbaulet A, Pötter R, Mazeron JJ et al. The GEC ESTRO Handbook of Brachytherapy. ESTRO, Brussels 2002.

9. Nag S. High dose rate brachytherapy: its clinical applications and treatment guidelines. Technol Cancer Res Treat 2004; 3: 269-287.

10. Ciérvide R, Ramos L, Aristu JJ et al. Use of customized-mould brachytherapy in the management of malignancies arising in the maxillary antrum after maxillectomy: a dosimetric analysis. Brachytherapy 2011; 10: 159-162.

11. Moretto D, Gargari M, Nordsjö E et al. Immediate loading: a new implant technique with immediate loading and aesthetics: Nobel ActiveTM. Oral Implantology 2008; 1: 50-55.

12. Mazeron JJ, Ardiet JM, Haie-Méder C et al. GEC-ESTRO recommendations for brachytherapy for head and neck squamous cell carcinomas. Radiother Oncol 2009; 91: 150-156.

13. Nishimura Y, Yoshihiko, Yokoe $Y$ et al. High-dose-rate brachytherapy using moulds for oral cavity cancer: The technique and its limitations. Int J Clin Oncol 1998; 3: 351-356.

14. Obinata $\mathrm{K}$, Ohmori $\mathrm{K}$, Shirato $\mathrm{H}$ et al. Experience of highdose-rate brachytherapy for head and neck cancer treated by a customized intra-oral mould technique. Radiat Med 2007; 25: 181-186.

15. Kudoh T, Ikushima H, Kudoh KT et al. High-dose-rate brachytherapy for patients with maxillary gingival carcinoma using a novel customized intraoral mould technique. Oral Surg Oral Med Oral Pathol Oral Radiol Endod 2010; 109: e102-108.

16. Chatani M, Tsuboi K, Yagi M et al. High dose rate brachytherapy using moulds after chemoradiotherapy for oral cavity cancer. Jpn J Radiol 2012; 30: 40-44.

17. Matsuzaki H, Takemoto M, Hara M et al. Two-piece customized mold technique for high-dose-rate brachytherapy on cancers of the buccal mucosa and lip. Oral Surg Oral Med Oral Pathol Oral Radiol 2012; 113: 118-125.

18. Budrukkar A, Dasgupta A, Pandit P et al. Clinical outcomes with high-dose-rate surface mould brachytherapy for intraoral and skin malignancies involving head and neck region. J Contemp Brachytherapy 2017; 9: 242-250.

19. Bhalavat R, Chandra M, Pareek V et al. High-dose-rate interstitial brachytherapy in head and neck cancer: do we need a look back into a forgotten art - a single institute experience. J Contemp Brachytherapy 2017; 9: 124-131.

20. Jones EL, Tonino Baldion, Thomas $C$ et al. Introduction of novel 3D-printed superficial applicators for high-dose rate skin brachytherapy. Brachytherapy 2017; 16: 409-414. 